\title{
Analysis of the Gene Expression and RNAi-Mediated Knockdown of Chitin Synthase from Leaf-Cutting Ant Atta sexdens
}

\author{
Ariele C. Moreira, ${ }^{a, b}$ Renato L. Carneiro, ${ }^{a}$ Mariana F. Fracola, ${ }^{a}$ Kelli C. Micocci, ${ }^{c}$ \\ Odair C. Bueno ${ }^{c}$ and Dulce H. F. Souza ${ }^{\circledR}, a$
}

\author{
${ }^{a}$ Departamento de Química, Universidade Federal de São Carlos, 13565-905 São Carlos-SP, Brazil \\ ${ }^{b}$ Departamento de Física, Química e Matemática, Universidade Federal de São Carlos, \\ 18052-780 Sorocaba-SP, Brazil \\ ${ }^{c}$ Centro de Estudos de Insetos Sociais, Universidade Estadual Paulista Júlio de Mesquita Filho, \\ 13506-900 Rio Claro-SP, Brazil
}

\begin{abstract}
Chitin synthase (CHS) is the enzyme specifically associated with chitin synthesis, an important component of diverse organisms including insects. Two alternative spliced transcripts of the CHS gene (AsCHS-A1 and AsCHS-A2) were identified by quantitative reverse-transcription polymerase chain reaction (RT-qPCR) during the development of the leaf-cutting ant Atta sexdens. Expression profiles of AsCHS-A transcripts increased from larva to pupae and decay in workers. Phylogenetic analysis showed both transcripts are classified within class A insect CHSs. AsCHS-A1 showed the highest expression level in larvae and pupae, while AsCHS-A2 is the main CHS transcript in workers. Our results suggest that these variants should be under regulation of different promoters. AsCHS-A1 has topology expected for insect CHSs, while the predicted AsCHS-A2 topology, with a missing A domain, is similar to some fungal CHSs. CHS-B (class B) was not identified in A. sexdens transcriptome. Ribonucleic acid interference (RNAi)-mediated gene silencing in pupae revealed that low reduction in CHS transcript levels (18\%) was enough to cause morphological changes in the pupa exoskeleton impairing the process of cuticle sclerotization. To our knowledge, this work was the first to use and to show the feasibility of using RNA interference techniques on leaf-cutting ants.
\end{abstract}

Keywords: leaf-cutting ant, chitin synthase (CHS), CHS gene expression, RNAi-mediated knockdown, CHS insect topology

\section{Introduction}

Chitin is a linear homopolymer of $\mathrm{N}$-acetyl$\beta$-D-glucosamine (GlcNAc) residues linked by $\beta$-1,4-glycosidic linkages. It is an important component of numerous organisms, mainly fungi (septum, spore and cell wall), arthropods (cuticle, peritrophic matrix, salivary gland, trachea, eggs and muscular attachment points), squids, mollusk shells and nematode eggshells. ${ }^{1-3}$

The insect cuticle has an inner layer of chitin filaments arranged within a protein matrix (procuticle) covered by the epicuticle, which consists of proteins and lipids. The cuticle is usually white when it is formed and becomes darker and more rigid during insect development due to stabilization through the incorporation of phenolic

*e-mail: dulce@ufscar.br compounds (sclerotization). ${ }^{4}$ Because the cuticle cannot expand, insect development is strictly dependent on the chitin biosynthesis. Therefore, any malfunction in the synthesis and/or degradation of chitin leads to developmental disorders, which may be observed during embryogenesis. . $^{2,5}$

Chitin synthesis is conserved from fungi to insects, and several enzymatic reactions are involved in the conversion of different sugar sources to GlcNAc polymers that are secreted to extracellular space, forming chitin microfibrils.? Chitin synthase (CHS) is the only enzyme class specifically associated with chitin synthesis, and it has been described as the key enzyme for this mechanism.,

Chitin synthases belong to the glycosyltransferase family 2 (GT-2), which includes other processive enzymes, such as cellulose synthases and hyaluronan synthases, among others. Enzymes of this class share two motifs: the 
aspartate-any residue-aspartate (DXD) motif of ribose/ metal $\mathrm{M}^{2+}$ coordination and the carboxylate residues (Asp-D or Glu-E) from donor and acceptor saccharide binding sites, which under physiological conditions are negatively loaded and act as the base of a nucleophilic substitution reaction, forming a glycosidic bond. ${ }^{5,8}$ Chitin synthase uses (UDP)-GlcNAc as an activated donor sugar (substrate), and requires divalent metal cations $\left(\mathrm{Mg}^{2+}\right.$ or $\mathrm{Mn}^{2+}$ ) to form the chitin polymer. ${ }^{5,9}$

To date, all insect genomes are known to contain one to two CHS genes, while fungi may have up to nine genes coding for CHSs. Insect CHSs are grouped into two classes of genes, class A (CHS-A or CHS-1) and class B (CHS-B or CHS-2). ${ }^{3,5,10}$ The two CHS classes have a similar structural organization and are comprised of three domains: $\mathrm{A}, \mathrm{B}$, and C. ${ }^{2}$ Domain A is found in the protein N-terminal and has low sequence similarity between different species, with the prediction of 7-10 transmembrane helices (TMHs). ${ }^{2}$ Domain B, a central domain of CHSs comprising approximately 400 amino acids, contains the catalytic region of the enzyme and it is oriented towards the cytoplasm. This domain includes six conserved GT-2 regions. Domain C corresponds to the enzyme C-terminal and contains seven TMHs in most insects CHSs, with the exception of fungal CHSs, which have three to five TMHs. In insects, the first two helices of domain $\mathrm{C}$ may undergo alternative splicing and the cluster of the other five TMHs was found to be involved in the extrusion of chitin polymers across the plasma membrane. , $^{2,11}$

Furthermore, domain $\mathrm{C}$ has two conserved regions: the $(\mathrm{S} / \mathrm{T}) \mathrm{WGT}(\mathrm{K} / \mathrm{R})$ and the coiled-coil motifs, the last one present only in class-A CHSs. The (S/T)WGT(K/R) motif belongs to a second conserved domain in fungal CHSs, essential to CHS activity toward synthesis of long chitooligosaccharide. ${ }^{12,13}$ The coiled-coil motif is predicted to be targeted towards the extracellular space and may serve as the site of protein-protein interactions involved in oligomerization. In addition, its position near the catalytic domain may aid in the regulation of chitin synthesis. ${ }^{5,10,14}$

Ants (Hymenoptera: Formicidae) represent one of the most diverse and ecologically dominant groups of eusocial insects in the world. Their emergence dates to 115-140 million years ago, and their presence in almost all habitats denotes their success in the terrestrial environment. ${ }^{15}$ The Attini tribe is composed of 45 genera, and 15 of them are fungus-growing ants that are dependent on the cultivation of fungi to feed the colony. Their geographic distribution is restricted to the American Continent, primarily the Neotropical region. ${ }^{16-18}$ Genera Atta and Acromyrmex represent the evolutionary apex of the tribe. They are known as leaf-cutting ants due to the large amounts of fresh plant material harvested for the cultivation of gardens of the symbiotic fungus. ${ }^{16}$ Leaf-cutting ants stand out for economic reasons in agriculture and forestry, since they cause significant annual losses; the effects may also be indirect, resulting from environmental contamination caused by the use of pesticides. ${ }^{19-21}$ Due to the absence of an efficient and safe control method, further studies on leaf ants physiology and behavior are essential.

In this study two open reading frame (ORF) of CHS (AsCHS-A1 and AsCHS-A2) from Atta sexdens have been identified. AsCHS-A1 and AsCHS-A2 are differentially regulated during insect development. AsCHS-A1 has topology pattern expected for insect CHSs while the predicted AsCHS-A2 topology was similar to some fungal CHSs. Gene expression analysis showed that CHS expression increased from larvae to pupae and decays in medium-sized workers. Functional analysis through silencing in pupae reveals that AsCHS is related to chitin synthesis in the ant cuticle and is important for ant development, once malfunction in the chitin synthesis leads to insect developmental disorder.

\section{Experimental}

\section{Biological samples}

Larvae, pupae and medium-sized workers of A. sexdens Forel (Hymenoptera: Formicidae) were collected from a laboratory nest at the Center for the Study of Social Insects (UNESP, Rio Claro, Brazil). The nest was supplied daily with Eucalyptus alba leaves and oat seeds, and occasionally with leaves of other plants such as Hibiscus sp. and Ligustrum sp. or rosebush petals. Samples were collected, washed with ribonuclease (RNase)-free phosphate-buffered saline (PBS) and stored at $-80{ }^{\circ} \mathrm{C}$ until ribonucleic acid (RNA) extraction.

\section{Identification of the AsCHS transcripts}

The total RNA was extracted from a pool of 10 samples for each developmental stage (10 larvae, 10 pupae and 10 workers) with TRIzol ${ }^{\circledR}$ (Thermo Fisher Scientific, Waltham, Massachusetts, USA) and PureLink ${ }^{\circledR}$ RNA mini Kit (Thermo Fisher Scientific, Waltham, Massachusetts, USA) as previously described. ${ }^{22}$ Total RNA $(1 \mu \mathrm{g})$ was treated with deoxyribonuclease (DNase, $1 \mathrm{U}$ ) (Thermo Fisher Scientific, Waltham, Massachusetts, USA) and first-strand complementary deoxyribonucleic acid (cDNA) was synthesized using SuperScript ${ }^{\circledR}$ VILO Master Mix 
(Thermo Fisher Scientific, Waltham, Massachusetts, USA) according to the manufacturer's instruction.

The cDNA from the three developmental stages was used as template for independent polymerase chain reaction (PCR). The identification of AsCHSs were initiated with a set of degenerate primers described in the literature ${ }^{14}$ to amplified both CHS-A and CHS-B of insects (Table 1). The amplified fragments were analyzed on a $1 \%$ agarose gel, purified using Wizard ${ }^{\circledR}$ SV Gel and PCR Clean-Up System (Promega, Madison, Wisconsin, USA) and sequenced on ABI 3730 DNA Analyzer (Thermo Fisher Scientific, Waltham, MA, USA).

New primers were designed based on the sequenced fragments to obtain full-length sequence of AsCHSs (Table 1, Figure 1).
cDNA and amino acid sequence analysis

The sequenced fragments were assembled using BioEdit. ${ }^{23}$ The homology search was carried out using the NCBI BLAST program. ${ }^{24}$ Amino acid sequence was deduced using Translate tool ${ }^{25}$ and the Sequence Manipulation Suite. ${ }^{26}$ Nucleotide and deduced amino acid sequence alignments were performed with Clustal Omega ${ }^{27}$ and MultiAlign. ${ }^{28}$ TMHMM $2.0^{29}$ and TOPCONS ${ }^{30}$ were used to topology prediction of transmembrane regions. Coiled-coils were predicted with Paircoil2. ${ }^{31}$ Promoter regions were identified with the Neural Network Promoter Prediction Tool. ${ }^{32}$ SignalP $4.1^{33}$ was used for signal peptides prediction.

Table 1. Nucleotide sequences of primers used in the AsCHSs identification

\begin{tabular}{|c|c|c|}
\hline Name & Sequence (5'-3') & Conserved aa sequence \\
\hline \multicolumn{3}{|c|}{ Degenerate primer } \\
\hline \multicolumn{3}{|c|}{ Forward primer } \\
\hline Fdeg & ATG DCG AAR AAY CAA CAT CAG & MTKNQHQ (MAKNQHQ) \\
\hline $\mathrm{F} 1^{\mathrm{a}}$ & TTY GAR TAY GCN ATH GGN CAY TGG & FEYAIGHW \\
\hline $\mathrm{F} \mathrm{a}^{\mathrm{a}}$ & TGY GCN ACN ATG TGG CAY G & CATMWHE \\
\hline $\mathrm{F}^{\mathrm{a}}$ & GGN TGG TGG GAR AA & GWWEN \\
\hline $\mathrm{F} 4^{\mathrm{a}}$ & CAR GAR ACN AAR GGN TGG GA & QETKGWE (ETKGW) \\
\hline $\mathrm{F}^{\mathrm{a}}$ & TGG GAY GTN TTY MGN GAY CCN CC & WDVFDDPP (WDVFR) \\
\hline \multicolumn{3}{|c|}{ Reverse primer } \\
\hline $\mathrm{R} 1^{\mathrm{a}}$ & CCA NCK RTC YTC NCC YTG RTC RTA YTG & QYDQGEDRW \\
\hline $\mathrm{R} 2^{\mathrm{a}}$ & ACY TCN CKN GTN CCC CA & WGTRE \\
\hline $\mathrm{R}^{\mathrm{a}}$ & AAN CKR TGR AAN ARC ATN GC & AMLFHRF \\
\hline $\mathrm{R} 4^{\mathrm{a}}$ & TTN GCN CCN TRN GTY TGC AT & MQTQ/YGAR (MQTLGA) \\
\hline Rdeg & TCA CAC GTT GGA GTT CRC CTC & EANSNVStop \\
\hline \multicolumn{3}{|c|}{ Specific primer } \\
\hline \multicolumn{3}{|c|}{ Forward primer } \\
\hline SF1 & CCC ATC TTG CTC TTC ATG & PILLFM \\
\hline SF2 & TAC AGC TTT TAT GCG CCC AGA TC & QLLCAQI \\
\hline SF3 & GCA ACA GCA TAA TGG CAA TGC & NSIMAM \\
\hline SF4 & GGT TTA ATC AGA TCG CTC GG & GLIRSL \\
\hline $\mathrm{SF} 2$ & CGT GCA AAT CGA ACA TAC AG & CKSNIQ \\
\hline F5UTR & GTG AAG TGG ATC CAG GAA ACG G & StopSGSRKR (frame 2) \\
\hline F_intron5 & GAA GTT CAG AGA AAG TCT ACA TC & EVQRKST \\
\hline \multicolumn{3}{|c|}{ Reverse primer } \\
\hline SR2 & AGC ACG CAG CCG ATC ATG TGT TCG & EHMIGCV \\
\hline SR3 & GCA TTG GTC CTC GTC CAG ACG CAG & LRLDEDQC \\
\hline SR4 & CCT CTC TAG ACA TTT CTG GTT TGC C & ANQKCLER \\
\hline R3UTR & CCT GGG GAG AAG CGC GAT G & IALLPR \\
\hline
\end{tabular}

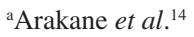


AsCHS-A1

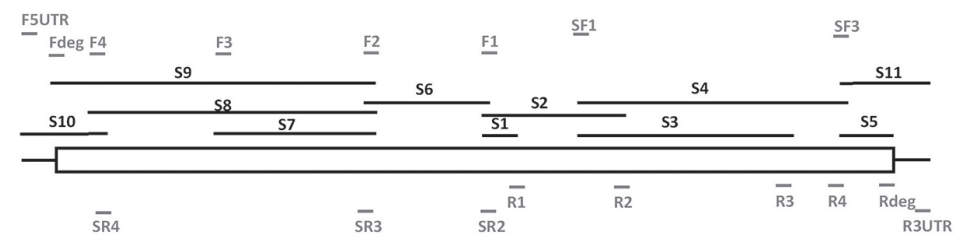

AsCHS-A2

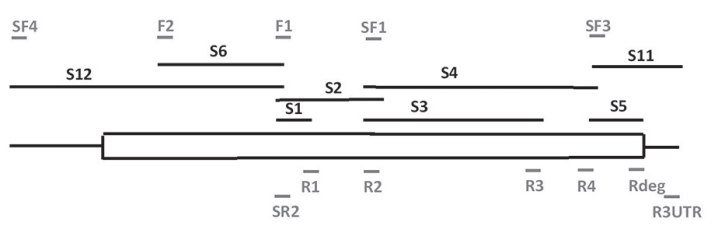

\begin{tabular}{lccc}
\hline & & AsCHS-A1 & AsCHS-A2 \\
\hline PCR fragment & Primers & \multicolumn{2}{c}{ Size(bp) } \\
\hline S1 & F1-R1 & 192 & 192 \\
S2 & F1-R2 & 857 & 567 \\
S3 & SF1-R3 & 1202 & 896 \\
S4 & SF1-R4 & 1595 & 1289 \\
S5 & SF3-Rdeg & 278 & 278 \\
S6 & F2-SR2 & 701 & 701 \\
S7 & F3-SR3 & 984 & --- \\
S8 & F4/F5-SR3 & 1683 & --- \\
S9 & Fdeg-SR3 & 1809 & --- \\
S10 & F5UTR-SR4 & 337 & --- \\
S11 & SF3-R3UTR & 437 & 437 \\
S12 & SF4-SR2 & --- & 1565 \\
\hline
\end{tabular}

Figure 1. Strategies for amplifying transcripts encoding chitin synthases in A. sexdens. Fragments identified in larvae and pupae (AsCHS-A1) and in medium-sized workers (AsCHS-A2) were amplified through multiple RT-PCR using the degenerate and/or specific primer combinations listed in the table. Lines (S1-S12) indicate amplified fragments; the box shows the ORF region and the solid lines at each end represent the 5'- and 3'-UTR regions.

\section{Phylogenetic analysis and classification of the AsCHSs}

Phylogenetic tree was constructed using MEGA version $7.0^{34}$ based on the neighbor-joining (NJ) method. For classification and phylogenetic analysis, both CHS-A and CHS-B from insects and fungal were included (details in Supplementary Information (SI) section).

\section{AsCHS expression analysis}

AsCHSs expression profile were evaluated using quantitative reverse-transcription PCR (RT-qPCR) of A. sexdens at different developmental stages ranging from larvae to adults. The experiment was carried out in biological triplicates for each sample. Total RNA was extracted as previously described. Total RNA was converted into cDNA in triplicate, and each cDNA was analyzed in three technical replicates. The same approach was used for all samples. The RT-qPCR was performed using the StepOnePlus ${ }^{\mathrm{TM}}$ system (Thermo Fisher Scientific, Waltham, MA, USA), with a 96-well plate sealed with MicroAmp optical adhesive film (Thermo Fisher Scientific,
Waltham, Massachusetts, USA). The final reaction volume was $12 \mu \mathrm{L}$, containing $6 \mu \mathrm{L}$ of Power SYBR ${ }^{\circledR}$ Green PCR Master Mix (Thermo Fisher Scientific, Waltham, Massachusetts, USA), $3 \mu \mathrm{L}$ of forward and reverse primers in adequate concentrations (Table 2; Figures S1 and S2, SI section) and $3 \mu \mathrm{L}$ of 100 -fold diluted cDNA.

The cDNA amplification conditions were as follows: $10 \mathrm{~min}$ at $95^{\circ} \mathrm{C}$ (polymerase activation) and then 40 cycles of $95{ }^{\circ} \mathrm{C}$ for $10 \mathrm{~s}$ (denaturation) and $60{ }^{\circ} \mathrm{C}$ for $1 \mathrm{~min}$ (annealing/extension). The dissociation curve of the PCR products (melting curve) was performed from 60 to $95{ }^{\circ} \mathrm{C}$ to ensure the specificity of the amplified product. The data were normalized to two stable reference genes during insect developments, efl-alpha and rplls, based on our previous evaluation ${ }^{22}$ (Table 2). The relative gene expression data were calculated using the $2^{-\Delta \Delta \mathrm{Ct}}$ method, ${ }^{35}$ data analysis was carried out on GraphPad Prism 5.0 $0^{36}$ and data are presented as mean \pm standard error of the mean (SEM). An analysis of variance (ANOVA) test was performed, followed by Mann-Whitney's post hoc tests for multiple comparisons; $P$ values $<0.05$ were considered significantly different.

Table 2. Sequences, optimum concentration and efficiency of the forward (F) and reverse (R) primers used in the RT-qPCR

\begin{tabular}{|c|c|c|c|c|}
\hline Primer name & $5^{\prime} \rightarrow 3^{\prime}$ sequence & $\mathrm{F} / \mathrm{R} / \mathrm{nM}$ & Product size / bp & Efficiency $^{\mathrm{a}} / \%$ \\
\hline pAsCHS & $\begin{array}{c}\text { F: CAAATTCTATTGATGGGTGGTACGA } \\
\text { R: GAAGGCACCCACCAACATG }\end{array}$ & $150 / 300$ & 69 & 97.4 \\
\hline pAsCHS-A1 & $\begin{array}{l}\text { F: GCGTACGCGATGATCATGAT } \\
\text { R: CGTCCTCGCCAAGCTGTAG }\end{array}$ & $100 / 100$ & 61 & 101.4 \\
\hline efl-alpha & $\begin{array}{c}\text { F: AGCCGCTGTTGCATTCGT } \\
\text { R: TGACGGATACTTCCAACATATTGTC }\end{array}$ & $100 / 150$ & 64 & 95.1 \\
\hline rplls & $\begin{array}{c}\text { F: CGAGATCATCACGTTCGATC } \\
\text { R: CTGCATCAAGACTGTACGTTTTCC }\end{array}$ & $150 / 300$ & 66 & 97.9 \\
\hline
\end{tabular}

${ }^{a}$ RT-qPCR efficiency calculated by standard curve method. pAsCHS: primer annealed to a common region of the AsCHS-A1 and AsCHS-A2 nucleotide sequences; pAsCHS-A1: primer annealed to a specific region of the AsCHS-A1 sequence; efl-alpha and rpl18: reference genes. ${ }^{22}$ 
Functional analysis of the AsCHSs using RNAi

The biological function of the AsCHS was analyzed through RNA interference (RNAi), injecting sequencespecific small interfering RNA (siRNA) into A. sexdens pupae. The siRNA nucleotide region common for both of AsCHSs was selected for siRNA synthesis (Figures 2 and 3). Region synthesized for AsCHS siRNA; sense: CCGUGGUAAGGCAUUGAUG[dT][dT]; antisense: CAUCAAUGCCUUACCACGG[dT][dT]. Universal siRNA negative control No. 1 (SIC001) (Sigma-Aldrich, St. Louis, MO, USA) and water were used as negative controls. The siRNAs or water were delivered into pupae by direct microinjection using a Nanoliter 2010 System with pulled borosilicate needles (World Precision Instruments, Sarasota, USA). The sample injected were AsCHS siRNA (300 ng/239.2 nL/pupa), control siRNA (300 ng/239.2 nL/ pupa) and water (239.2 nL/pupa). Artificial micronests were made in Petri dishes containing fungus garden and medium-sized workers. Prior to the introduction of the injected pupae the micronests were kept dormant at $-20{ }^{\circ} \mathrm{C}$ for $30 \mathrm{~min}$ and a sucrose solution was sprayed over each micronest and after that ten injected pupae were added to each micronest. For each experimental group five micronests were used.

Analyses of the phenotype and mRNA-level were performed 24, 48, 72, 168 and $240 \mathrm{~h}$ post-injection. The experiments were performed in biological duplicate.

\section{Results and Discussion}

\section{Identification and characterization of the AsCHSs}

The genome of $A$. sexdens has not been sequenced yet. Therefore, CHSs coding sequences were determined through multiple PCR amplifications using degenerate primers based on conserved regions of insect CHSs, and specific primers from 5' - and 3'-UTR (untranslated region) of the other ants with a sequenced genome (Table 1). Two transcripts of AsCHS-A in the different developmental



Figure 2. Alignment of the amino acid residues of AsCHS-A1 and AsCHS-A2. Conserved regions of insect CHSs that were used to design the primers in RT-PCR are shaded in green. The coiled-coil motif is underlined by a single blue line and the conserved regions of the catalytic domain are enclosed in a red box (1-6). 
(a)

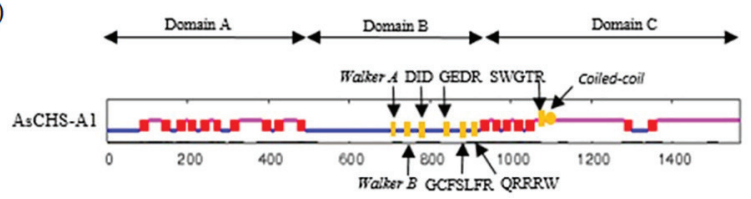

(b)

(c)

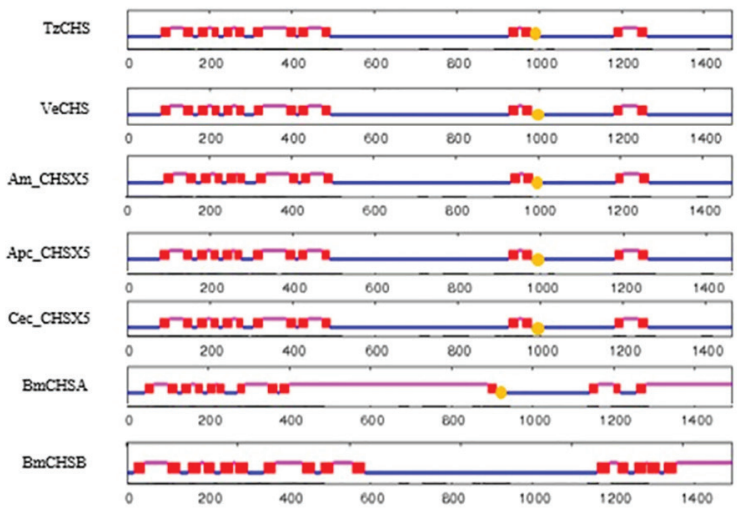

Figure 3. Topology predictions for insect chitin synthases. (a) Topology prediction for AsCHS-A1; (b) topology prediction for AsCHS-A2; (c) other insect CHSs (B. mori [BmCHSA (AFB83705.1) and BmCHSB (AFC69002.1)], predicted CHS sequences for T. zeteki [TzCHS (KYQ52765.1)], V. emeryi [VeCHS (011866715.1)], A. mellifera isoform X5 [Am_CHSX5 (XP_016770740.1)], A. cerana isoform X5 [Apc_CHSX5 (XP_016906999.1)], and C. cinctus isoform X5 [Cec_CHSX5 (XP_015608867.1)]). The three domains, A, B, and C are indicated at the top. Vertical red bars represent the transmembrane region, the horizontal blue line depict intracellular (down line) and the extracellular domains by pink line (top line). Conserved regions and the coiled-coil motif are indicated by yellow boxes and a circle, respectively.

stages were identified. The 11 fragments identified in larvae and pupae led to the same sequence called AsCHS-A1 (GenBank accession number MH934187), while the 8 fragments from medium-sized workers resulted another sequence, AsCHS-A2 (GenBank accession number MH934188). The combination of primers used in the PCR and the sizes of the amplified fragments are shown in Figure 1.

The complete cDNA sequence of AsCHS-A1 is $4987 \mathrm{bp}$. The ORF of AsCHS-A1 is $4707 \mathrm{bp}$ and encode a polypeptide of 1568 amino acid residues, with no prediction of signal peptides. The AsCHS-A2 is $3696 \mathrm{bp}$, an ORF of $2877 \mathrm{bp}$ coding for 958 amino acid residues with no predicted signal peptide. Based on deduced amino acid sequence, AsCHS-A1 and AsCHS-A2 enzymes were classified as CHS-coding sequences due to the presence of two signature motifs in the GT-2 family, EDR and QRRRW. The alignment of the deduced amino acid sequences of the variant transcripts AsCHS-A1 and AsCHS-A2 are shown in Figure 2. The differential exon usage result in AsCHS-A2 absence of 508 amino acids in the $\mathrm{N}$-terminal region and 102 amino acids in the middle of the sequence, including the deletion of the (S/T)WGT(K/R) motif when compared to AsCHS-A1.

\section{AsCHS-A1 and AsCHS-A2 topology predictions}

The predictions for TMHs using the software TMHMM 2.0 ${ }^{29}$ suggested that AsCHS-A1 and AsCHS-A2 are membrane associated proteins. The topology for AsCHS-A1 (Figure 3a) included three predicted domains similar to other known insect CHS proteins. The domain A has ten TMHs, the domain B corresponding to the highly conserved catalytic domain in the GT-2 family of glycosyltransferases, turned towards the intracellular medium. The domain C included seven TMHs, the SWGTR motif, another signature motif, and a coiled-coil motif, both toward to extracellular space.

The predicted topology for the AsCHS-A2 shows only two of the three domains normally found in insect CHSs (Figure $3 \mathrm{~b}$ ), the $\mathrm{B}$ and $\mathrm{C}$ domains. Conserved regions of the N-terminus (such as GWWEN, ETKGW, WDVFR and WDVFDDPP, usually present in insect CHSs) and the 5'-UTR region identified in AsCHS-A1 could not be amplified, despite the use of specific and degenerate primers (Table 1). To our knowledge, to date there are no descriptions of insect CHSs without domain A, as found here for medium-sized workers. However, the complete absence of the domain A was described for some classes of fungal CHSs, which have no transmembrane regions and are smaller in size compared to insect proteins, as discussed by Merzendorfer. ${ }^{7}$ Further, the deletion of domain A in class I and II fungi (389 and 221 amino acid residues, respectively) did not lead to loss of enzyme activity or function. ${ }^{37}$ After the action of trypsin in fungal CHS the enzymatic activity was not affected by the release of fragments on the N-terminal and even for truncated enzyme in this portion, showing that the active site is sufficient for the catalytic activity. ${ }^{38}$ These results show that domain $\mathrm{A}$ is probably not essential for the catalytic activity of the enzyme.

Domain B comprises the catalytic domain, is highly conserved and is identical to both AsCHS-A1 and AsCHS-A2 (Figure 3c). Six highly conserved regions of the GT-2 family are present: (1) GKTKMIAHLKDKSKIRHRKR and (2) KEVIAENTFLLTLD, both involved in UDP-binding sites; (3) DID and (4) GCFSLFR, both acceptor saccharide binding sites; (5) GEDR, an acceptor saccharide-binding site; and (6) QRRRW, a product-binding site. Regions 5 and 6 have been described as GT-2 consensus regions and are essential for the catalytic activity. ${ }^{2,5,12}$ Thus, it may be inferred that the enzymatic function is maintained in both 
identified enzymes. In accordance, recent advance on understanding of translocation of the cellulose shows that the translocation mechanism takes place due to the finger helix, in insect this region was identified as cointaining the highly conserved EDR motif. The up and down movement of the finger helix to push the polymer into the channel was also confirmed for fungal chitin synthase and is essential to polymer synthesis. ${ }^{39,40}$

Domain $\mathrm{C}$ of AsCHS-A2 has a different TMH pattern when compared to AsCHS-A1, including four TMHs, a coiled-coil motif (in the intracellular medium) and the absence of the (S/T)WGT(K/R) motif. The similarity search found six other CHS insect sequences that share this pattern with AsCHS-A2 (Figure 3c). Of these, five are predicted sequences belonging to the Hymenoptera order, two in family Formicidae (Trachymyrmex zeteki, Vollenhovia emeryi), two in family Apidae (Apis cerana, Apis mellifera), one in family Cephidae (Cephus cinctus) and one in Lepidoptera order (Bombyx mori). Of these insects, only B. mori has been studied for gene organization, presence of alternative splicing, deletion of the $(\mathrm{S} / \mathrm{T}) \mathrm{WGT}(\mathrm{K} / \mathrm{R})$ motif and gene expression, ${ }^{41-43}$ with the conclusion that, although alternative splicing in this region lead to changes in insects TMH predictions, this is a functional enzyme.

\section{Analysis of alternative splicing of the AsCHSs}

The occurrence of alternative splicing in domain $\mathrm{C}$ is predicted for insect CHSs and is characteristic of class-A CHS, since it was not identified in class-B CHS. ${ }^{14}$ Sequence analysis showed that AsCHS-A1 and AsCHS-A2 share a higher identity with insect CHS-A (71-98\%) than with insect CHS-B (44-57\%) (data not shown). CHS-A is responsible for chitin synthesis in the epidermis and in ectodermal cells, such as trachea, egg and ovary cells, ${ }^{14,44,45}$ while the CHS-B gene is involved in chitin synthesis in intestinal epithelial cells, such as the peritrophic membrane..$^{2,14,44}$

The AsCHS-A1 and AsCHS-A2 sequences were compared to the CHS gene of T. zeteki (Tz_CHS8; GenBank KQ982655.1), which also belongs to tribe Attini. The Tz_CHS8 gene contains 20808 bp, including 18 exons and 17 introns and a 4407-bp ORF (GenBank KYQ52765.1) (Figure 4a). The alignment between AsCHS-A1, AsCHS-A2, and Tz_CHS8 revealed that the exons of AsCHS were very similar to those found in Tz_CHS8 (Figures 4b and 4c). The 5'-UTR region of AsCHS-A 1 should be interrupted by a $9.2 \mathrm{~kb}$ intron, a similar pattern was found in Ostrinia furnacalis CHS-A (OfCHSA-2a). ${ }^{46}$ Exon $12 \mathrm{~b}$ is present in AsCHS-A1 transcripts but absent from transcripts of Tz_CHS and AsCHS-A2 (Figure 4). The presence of this sequence in the CHS genome of T. zeteki confirms the hypothesis of alternative splicing in domain $\mathrm{C}$.

The absence of the exon 2 and the exon 4 in AsCHS-A2 was confirmed by non-amplification by PCR of S8 and S7 fragments, respectively, using medium-sized worker cDNA as template in PCR and the combination of primers (Figure 1). The difference in the 5'-UTR region in AsCHS-A2 was confirmed by non-amplification of S10 fragment using F5UTR-SR4 or F5UTR-SR3 primers (Figure 1). Modifications in 5'-UTR regions of AsCHS-A1 and AsCHS-A2 (Figures $4 \mathrm{~b}$ and $4 \mathrm{c}$ ) suggest that, although both AsCHS sequences are encoded by the same gene, they are probably regulated by different promoters, as described for O. furnacalis $^{46}$ and B. mori. ${ }^{41}$ Other experiments need to be carried out to confirm this hypothesis.

Only two CHS genes have been described for insects, but studies ${ }^{14}$ show that different isoforms result from alternative splicing of CHS-A, which appears to

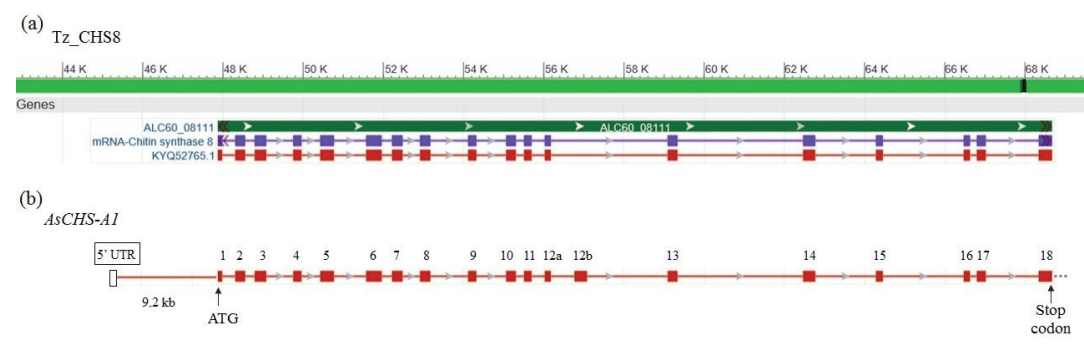

(c)

ASCHS-A2

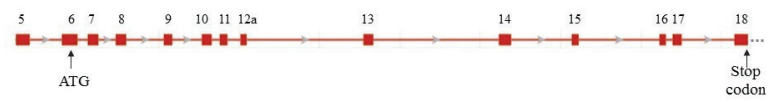

Figure 4. Comparison of the genomic structure of AsCHS to the genome of T. zeteki. (a) Representation of the structure of the Tz_CHS8 gene. The gDNA is presented in dark green, the coding region is blue, and the mRNA is red (available online at NCBI under accession number KQ982655.1); (b) predicted structure for AsCHS-A1; (c) predicted structure for AsCHS-A2. Red solid boxes indicate exons of the ORF, white boxes the 5'-UTR region, the dashed line the 3'-UTR region, the solid line corresponds to introns, start (ATG) and stop codons obtained are shown. 
be regulated during insect development. Two splice variants of CHS were reported for insect species, including Anopheles gambiae, ${ }^{47}$ Bactrocera dorsali, ${ }^{48}$ Locusta migratoria manilensis, ${ }^{49}$ Manduca sexta, ${ }^{50}$ Nilaparvata lugens and Laodelphax striatellus, ${ }^{51}$ Plutella xylostella, ${ }^{52}$ Spodoptera exigua ${ }^{53}$ and Tribolium castaneum. ${ }^{14}$ In these sequences, the position of the splice may vary, however, all are in the domain $\mathrm{C}$ of the CHSs and usually include the first and the second transmembrane regions after the catalytic domain. These studies have shown that CHS-Aa and CHS-Ab are predominantly expressed in the integument and in the trachea, respectively.

Recently, up to four CHS isoforms have been described in Lepidoptera order insects, such as $O$. furnacalis $^{46}$ and B. $m o r i{ }^{41}$ Two promoters were identified in these insects, one located upstream of the 5'-UTR region and the other located between exons $2 \mathrm{a}$ and $2 \mathrm{~b}$. Domain $\mathrm{A}$ of the transcripts of the CHSA-2a comprises exons 1, 2a, and 3 , and the transcripts of the CHSA- $2 b$ include exons $2 b$ and 3 , but not exon $1{ }^{41,46}$ Similarly, A. sexdens should have a different promoter controlling expression and providing variant transcripts of AsCHS-A. The transcripts identified of AsCHS-A2 starts at exon 5. Our analysis suggest that the translation of this enzyme should be starting in initiator ATG present in exon 6 although other experiments need to be done to confirm this hypothesis, such as 5'-RACE.

In this work, CHS-B was not identified in larvae, pupae and medium-sized workers of the A. sexdens transcriptome. The peritrophic matrix consists basically of chitin and proteins, has a variable rate of formation, and is stimulated during the feeding stages of insects. ${ }^{54}$ There are evidences that some insects do not have a peritrophic matrix, such as the adults of some ant species (Hymenoptera). ${ }^{55}$ CHS-B transcripts would be expected at least in the larval and medium-sized worker stages, the feeding stages of ants. In A. sexdens ants, the larvae feed almost exclusively on staphylae (97.5\%), which are a set of swollen hyphal tips (gongylidia) produced by the fungus garden. These structures are filled with liquid containing higher concentrations of lipids and carbohydrates than the rest of the fungus. ${ }^{56,57}$ Ant workers only ingest liquid food, partly derived from the preparation of staphylae for larvae and from proctodeal trophallaxis with the larvae. ${ }^{56}$ This behavior of the leaf-cutting ants could explain why CHS-B transcripts were not found. Insects of the order Hemiptera (Anasa tristis, Acyrthosiphon pisum, Nilaparvata lugens, and Rhodnius prolixus) are the only insects described as having a single CHS gene instead of two. These insects do not have a true peritrophic matrix and may have lost one of the CHS genes during evolution. ${ }^{51,58,59}$

\section{Phylogenetic analysis}

The phylogenetic tree was constructed based on amino acid sequences of AsCHS-A1, AsCHS-A2, insects and fungus CHSs from class A and class B. The analysis included insects of order Hymenoptera, A. mellifera, and the predicted CHS sequences for ants. From ants, only Atta cephalotes and A. colombica had CHS sequences belonging to both class A and class B. Genomic analysis of these ants confirmed the presence of two genes responsible for CHS synthesis (A. cephalotes: LOC105621766 and LOC105621961; and A. colombica: LOC108690240 and LOC108691753), but there are no studies of their transcriptome. The phylogenetic tree (Figure S3, SI section) showed that AsCHS-A1 and AsCHS-A2 were related to Hymenoptera and related to the class-A CHS proteins from other insects, confirming their identity as members of class-A CHSs, as predicted based on the presence of a coiled-coil and alternative splicing. AsCHS-A1 and AsCHS-A2 should be related to chitin synthesis in ant cuticles. They are not from endosymbiont fungi, also included in the phylogenetic tree.

\section{AsCHS expression analysis}

Gene expression profiles of $A$. sexdens CHS spliced transcripts during developmental stages were performed by RT-qPCR. The pAsCHS primer annealed to a common region of the variant transcripts AsCHS-A1 and AsCHS-A2 (hereafter called total transcripts) and the pAsCHS-A1 primer annealed to a specific region of the AsCHS-A1, quantifying only AsCHS-A1 transcripts. Figures S1 and S2 (SI section) show the AsCHS-A1 and AsCHS-A2 nucleotides sequences and the regions of the pAsCHS and pAsCHS-A1 primers.

Total transcripts of AsCHS increased from larvae to pupae and decreased in medium-sized worker, the last with the lowest level of expression (Figure 5A). This pattern of AsCHSs expression is associated with molting cycle and may be associated with the requirement of chitin synthesis in the epidermis, confirming their classification as CHS-A. At the adult stage, there is no molting and the insect has already reached its maximum size, so there is no need to synthesize chitin for ecdysis, which is consistent with the lower CHS expression level in medium-sized workers. The lower CHS expression at the adult stage was also observed in other insects, such as T. castaneum,,$^{14}$ L. migratoria manilensis, ${ }^{49}$ Rhodnius prolixus, ${ }^{58}$ and $O$. furnacali ${ }^{46}$

Although it was not possible to amplify AsCHS-A1 in medium-sized workers using conventional PCR, AsCHS-A1 transcript was observed by RT-qPCR (Figure 5B). The 

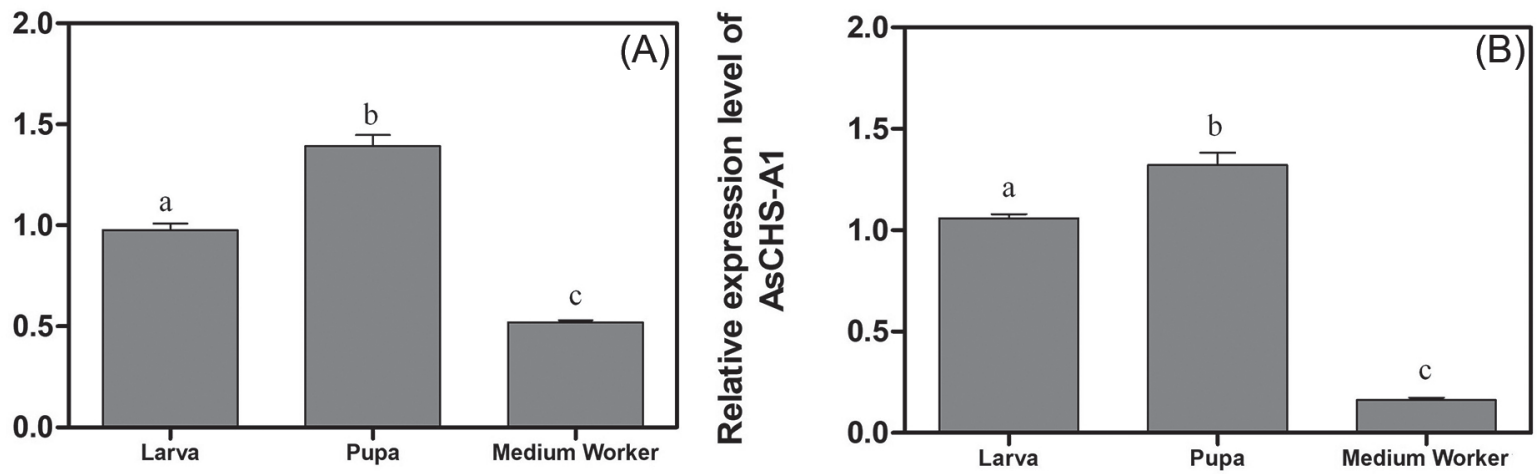

Figure 5. Analysis of the gene expression of AsCHS at three developmental stages of A. sexdens. (A) Relative expression level of total AsCHS transcript at each developmental stage; (B) relative expression level of AsCHS-A1 expression at each developmental stage. The relative expression level of AsCHSs were measured using references genes (efl-alpha and rpl18) and are relative to the larval stage. Values are mean \pm SEM. Statistical analysis with ANOVA and multiple comparison by post hoc Mann-Whitney's tests $(P<0.05)$; significant differences are indicated by different letters.

relative expression level for this developmental stage reveals total transcripts of AsCHS almost 22-fold higher than AsCHS-A1 (data not shown), showing that AsCH-A2 should correspond to the predominant transcript in mediumsized worker. Beside this, the AsCHS-A1 is the main CHS transcript in larvae and pupae.

\section{RNAi-mediated silencing of the AsCHS gene}

The effect of RNAi-mediated silencing of the AsCHS gene was assessed in pupae with two control groups injected with water or control siRNA. The selective decrease of target gene expression was evaluated after pupae injection. The RT-qPCR analysis indicated that pupae injected with $300 \mathrm{ng}$ of AsCHS siRNA had an 18\% reduction in total transcript levels of AsCHS after $168 \mathrm{~h}$ compared to the control groups (Figure 6) and reached normal levels $240 \mathrm{~h}$ after the injection (Figure S4, SI section).



Figure 6. Relative gene expression analysis of total AsCHS transcript levels $168 \mathrm{~h}$ (7 days) after the injection of $300 \mathrm{ng}$ of siRNA in pupae. Pupae and controls were injected with AsCHS siRNA and control siRNA and water, respectively. The relative expression level of AsCHSs were measured using references genes (efl-alpha and rpll8) and are relative to water control. Values are mean \pm SD. Statistical analysis with multiple comparison by Mann-Whitney's tests $(P<0.05)$; significant differences are indicated by different letters.
Injection of RpCHS-1 double-stranded RNA (dsRNA) in nymphs of $R$. prolixus caused 50\% down-regulation in CHS-1 transcript levels resulting in lethal phenotypes after 15 days of treatment. ${ }^{58}$ In L. migratoria manilensis nymphs, treatment with LmCHS1 dsRNA led to abnormal phenotypes with incomplete ecdysis and an approximately $80 \%$ reduction in LmCHS1 transcripts levels. ${ }^{49}$ Other insects treated with CHS-A dsRNA also showed alterations in phenotype and ecdysis, such as T. castaneum, ${ }^{44}$ S. exigua ${ }^{60}$ and B. dorsalis. ${ }^{48}$

In the present study, morphological changes in the exoskeleton of the A. sexdens gaster were observed $168 \mathrm{~h}$ post-injection (Table 3). These changes consisted of shrinkage, poor formation of the gastric region and presence of lighter regions in the cuticle, while the two control groups had a homogeneous cuticle. The presence of a dark dot on the gaster of some of the treated pupae indicates the injection site. After $240 \mathrm{~h}$ the color of the AsCHS siRNA-treated cuticle of the pupae was lighter, suggesting that the sclerotization process may have been impaired (Table 3). This result indicates a lower level of stabilization of the cuticle, which may be associated with the difficulty of incorporating the phenolic compounds necessary for cuticle sclerotization. The incorporation of these compounds occurs both through cuticular proteins and through covalent bonds to chitin structures. ${ }^{4}$ Therefore, our results were significant for RNAi-mediated silencing of the AsCHS gene when we analyzed the pupae phenotypes. According to Wang et al. ${ }^{61}$ in their studies with Tribolium castaneum and siRNAASH (achaete-scutehomolog) have shown that siASH-1 could depress Tc-ASH expression by around $45 \%$, but only in a short period (from day 2 to day 4) and it did not trigger any phenotypic change. Our results were less efficient when compared with the Wang et al ${ }^{61}$ in the suppression of the target transcript, but the deformation of the pupae was much higher than in Tribolium. 
Table 3. Phenotypic analysis of A. sexdens pupae after the injection of $300 \mathrm{ng}$ of siRNA. Pupae and negative controls were injected with AsCHS siRNA and control siRNA and water, respectively. The analysis was performed 168 and $240 \mathrm{~h}$ post-injection; black arrows indicate morphological changes in the gaster of pupae



siRNA: small interfering RNA; AsCHS: chitin synthase from the leaf-cutting ant Atta sexdens.

\section{Conclusions}

In conclusion, two alternative spliced exon of CHS gene from A. sexdens were identified and characterized. The variants transcripts should be regulated by different promoters during insect development. The enzymes sequences, deduced from the transcripts, were classified as CHS-A. AsCHS had a higher level of expression in larvae and pupae than in workers. Silencing AsCHS in pupae through the injection of siRNA resulted in a small decrease in CHS expression, however, this down-regulation was enough to cause morphological modifications of the gaster exoskeleton region of pupae and impair the cuticle stabilization even after reaching normal levels of CHS, showing that malfunction in the chitin synthesis leads to developmental disorder. To our knowledge, this work was the first to use and to show the feasibility of using RNA interference techniques on leaf-cutting ants. This method was used for the genomic analysis of the CHS function, but it is also promising for the evaluation of other potential targets for the control of leaf-cutting ants.

\section{Supplementary Information}

Supplementary information (nucleotides and deduced amino acid sequences of AsCHS-A1 and AsCHS-A2 amplified using the larvae and pupae cDNA of A. sexdens and phylogenetic tree of CHSs from insects and fungi) is available free of charge at http://jbcs.sbq.org.br as PDF file. 


\section{Acknowledgments}

This work was supported by grant No. 160662/2013-3 from CNPq and grants No. 2014/12169-2, 2012/25299-6 and 2017/06198-8 from the São Paulo Research Foundation (FAPESP). This study was financed in part by the Coordenação de Aperfeiçoamento de Pessoal de Nível Superior, Brazil (CAPES), finance code 001.

\section{Author Contributions}

Dulce H. F. Souza and Odair C. Bueno designed the research and provided guidance; Renato L. Carneiro and Ariele C. Moreira performed the statistical analysis; Ariele C. Moreira, Mariana F. Fracola and Kelli C. Micocci performed the experiments; Dulce H. F. Souza and Ariele C. Moreira wrote the manuscript. All authors read and approved the final manuscript.

\section{References}

1. Merzendorfer, H.; Insect Sci. 2013, 20, 121.

2. Merzendorfer, H.; Zimoch, L.; J. Exp. Biol. 2003, 206, 4393.

3. Muthukrishnan, S.; Merzendorfer, H.; Arakane, Y.; Kramer, K. J. In Insect Molecular Biology and Biochemistry, ${ }^{\text {st }}$ ed.; Gilbert, L. I., ed.; Academic Press: London, United Kingdom, 2012, p. 193.

4. Andersen, S. O.; Insect Biochem. Mol. Biol. 2010, 40, 166.

5. Merzendorfer, H.; J. Comp. Physiol., B 2006, 176, 1.

6. Zhu, K. Y.; Merzendorfer, H.; Zhang, W.; Zhang, J.; Muthukrishnan, S.; Annu. Rev. Entomol. 2016, 61, 177.

7. Merzendorfer, H.; Eur. J. Cell Biol. 2011, 90, 759.

8. Coutinho, P. M.; Deleury, E.; Davies, G. J.; Henrissat, B.; J. Mol. Biol. 2003, 328, 307.

9. Glaser, L.; Brown, D.; J. Biol. Chem. 1957, 228, 729.

10. Zhu, Y. C.; Specht, C. A.; Dittmer, N. T.; Muthukrishnan, S.; Kanost, M. R.; Kramer, K. J.; Insect Biochem. Mol. Biol. 2002, 32, 1497.

11. Richmond, T.; Genome Biol. 2000, 1, reviews3001.1.

12. Dorfmueller, H. C.; Ferenbach, A. T.; Borodkin, V. S.; van Aalten, D. M. F.; J. Biol. Chem. 2014, 289, 23020.

13. Yabe, T.; Yamada-Okabe, T.; Nakajima, T.; Sudoh, M.; Arisawa, M.; Yamada-Okabe, H.; Eur. J. Biochem. 1998, 258, 941.

14. Arakane, Y.; Hogenkamp, D. G.; Zhu, Y. C.; Kramer, K. J.; Specht, C. A.; Beeman, R. W.; Kanost, M. R.; Muthukrishnan, S.; Insect Biochem. Mol. Biol. 2004, 34, 291.

15. Brady, S. G.; Schultz, T. R.; Fisher, B. L.; Ward, P. S.; Proc. Natl. Acad. Sci. U. S. A. 2006, 103, 18172.

16. Hölldobler, E.; Wilson, E. O.; The Ants, $1^{\text {st }}$ ed.; Harvard University Press: Cambridge, MA, USA, 1990.

17. Ward, P. S.; Brady, S. G.; Fisher, B. L.; Schultz, T. R.; Syst. Entomol. 2015, 40, 61.
18. Weber, N. A.; Gardening Ants: The Attines, $1^{\text {st }}$ ed.; American Philosophical Society: Philadelphia, USA, 1972.

19. Britto, J. S.; Forti, L. C.; Oliveira, M. A.; Zanetti, R.; Wilcken, C. F.; Zanuncio, J.; Loeck, A. E.; Caldato, N.; Nagamoto, N. S.; Lemes, P. G.; Camargo, R. S.; Int. J. Res. Environ. Stud. 2016, 3, 11.

20. Della Lucia, T. M. C.; Fowler, H. G. In As Formigas Cortadeiras, $1^{\text {st }}$ ed.; Della Lucia, T. M. C., ed.; Folha de Viçosa: Viçosa, Brazil, 1993.

21. Della Lucia, T. M. C.; Souza, D. J. In Formigas-Cortadeiras: da Biologia ao Manejo, $1^{\text {st }}$ ed.; Della Lucia, T. M. C., ed.; UFV: Viçosa, Brazil, 2011.

22. Moreira, A. C.; Santos, A. M.; Carneiro, R. L.; Bueno, O. C.; Souza, D. H. F.; Int. J. Environ. Agric. Biotechnol. 2017, $2,743$.

23. Hall, T. A.; Bull. Biosci. 2011, 2, 60.

24. https://blast.ncbi.nlm.nih.gov/Blast.cgi, accessed in May 2020.

25. http://web.expasy.org/translate/, accessed in May 2020.

26. https://www.bioinformatics.org/sms/show_trans.html, accessed in May 2020.

27. https://www.ebi.ac.uk/Tools/msa/clustalo/, accessed in May 2020.

28. Corpet, F.; Nucleic Acids Res. 1988, 16, 10881.

29. http://www.cbs.dtu.dk/services/TMHMM/, accessed in May 2020.

30. http://topcons.cbr.su.se/, accessed in May 2020.

31. http://cb.csail.mit.edu/cb/paircoil2/paircoil2.html, accessed in May 2020.

32. http://www.fruitfly.org/seq_tools/promoter.html, accessed in May 2020.

33. Petersen, T. N.; Brunak, S.; von Heijne, G.; Nielsen, H.; Nat. Methods 2011, 8, 785.

34. Kumar, S.; Stecher, G.; Tamura, K.; Mol. Biol. Evol. 2016, 33 , 1870.

35. Livak, K. J.; Schmittgen, T. D.; Methods 2001, 25, 402.

36. Motulsky, H. J.; Prism 5 Statistics Guide, GraphPad Software Inc., San Diego CA, USA, 2007.

37. Cabib, E.; Shaw, J. A.; Mol, P. C.; Bowers, B.; Choi, W.-J. In The Mycota: A Comprehensive Treatise on Fungi as Experimental Systems for Basic and Applied Research, $1^{\text {st }}$ ed.; Esser, K., ed.; Springer: Heidelberg, Berlin, Germany, 1996.

38. Uchida, Y.; Shimmi, O.; Sudoh, M.; Arisawa, M.; YamadaOkabe, H.; J. Biochem. 1996, 119, 659.

39. Gohlke, S.; Muthukrishnan, S.; Merzendorfer, H.; Int. J. Mol. Sci. 2017, 18, 702.

40. Morgan, J. L. W.; Mcnamara, J. T.; Fischer, M.; Rich, J.; Withers, S. G.; Zimmer, J.; Nature 2016, 531, 329.

41. Xu, G.; Zhang, J.; Liu, J.; Ding, Y.; Feng, Q.; Song, Q.; Zheng, S.; Insect Biochem. Mol. Biol. 2017, 87, 117.

42. Zhuo, W.; Chu, F.; Kong, L.; Tao, H.; Sima, Y.; Xu, S.; Arch. Insect Biochem. Physiol. 2014, 85, 36.

43. Zhuo, W.; Fang, Y.; Kong, L.; Li, X.; Sima, Y.; Xu, S.; Mol. Biol. Rep. 2014, 41, 4177. 
44. Arakane, Y.; Muthukrishnan, S.; Kramer, K. J.; Specht, C. A.; Tomoyasu, Y.; Lorenzen, M. D.; Kanost, M.; Beeman, R. W.; Insect Mol. Biol. 2005, 14, 453.

45. Arakane, Y.; Specht, C. A.; Kramer, K. J.; Muthukrishnan, S.; Beeman, R. W.; Insect Biochem. Mol. Biol. 2008, 38, 959.

46. Qu, M.; Yang, Q.; Insect Biochem. Mol. Biol. 2011, 41, 923.

47. Zhang, X.; Zhang, J.; Park, Y.; Zhu, K. Y.; Insect Biochem. Mol. Biol. 2012, 42, 674.

48. Yang, W.-J.; Xu, K.-K.; Cong, L.; Wang, J.-J.; Int. J. Biol. Sci. 2013, 9, 331.

49. Zhang, J.; Liu, X.; Zhang, J.; Li, D.; Sun, Y.; Guo, Y.; Ma, E.; Zhu, K. Y.; Insect Biochem. Mol. Biol. 2010, 40, 824.

50. Hogenkamp, D. G.; Arakane, Y.; Zimoch, L.; Merzendorfer, H.; Kramer, K. J.; Beeman, R. W.; Kanost, M. R.; Specht, C. A.; Muthukrishnan, S.; Insect Biochem. Mol. Biol. 2005, 35, 529.

51. Wang, Y.; Fan, H. W.; Huang, H. J.; Xue, J.; Wu, W. J.; Bao, Y. Y.; Xu, H. J.; Zhu, Z. R.; Cheng, J. A.; Zhang, C. X.; Insect Biochem. Mol. Biol. 2012, 42, 637.

52. Ashfaq, M.; Sonoda, S.; Tsumuki, H.; Pestic. Biochem. Physiol. 2007, 89, 20.

53. Chen, X.; Yang, X.; Kumar, N. S.; Tang, B.; Sun, X.; Qiu, X.; Hu, J.; Zhang, W.; Insect Biochem. Mol. Biol. 2007, 37, 409.

54. Lehane, M. J.; Annu. Rev. Entomol. 1997, 42, 525.
55. Peters, W.; Peritrophic Membranes; Springer-Verlag: Berlin, Germany, 1992.

56. Schneider, M. O.: Comportamento de Cuidado da Prole da Saúva-Limão Atta sexdens rubropilosa FOREL, 1908 (HYMENOPTERA, FORMICIDAE); MSc dissertation, UNESP, Rio Claro, Brazil, 2003, available at https://repositorio.unesp. br/bitstream/handle/11449/88529/schneider_mo_me_rcla. pdf?sequence=1, accessed in May 2020.

57. Schiøtt, M.; Rogowska-Wrzesinska, A.; Roepstorff, P.; Boomsma, J. J.; BMC Biol. 2010, 8, 156.

58. Mansur, J. F.; Alvarenga, E. S. L.; Figueira-Mansur, J.; Franco, T. A.; Ramos, I. B.; Masuda, H.; Melo, A. C. A.; Moreira, M. F.; Insect Biochem. Mol. Biol. 2014, 51, 110.

59. The International Aphid Genomics Consortium; PLoS Biol. 2018, 16, e3000029.

60. Tian, H.; Peng, H.; Yao, Q.; Chen, H.; Xie, Q.; Tang, B.; Zhang, W.; PLoS One 2009, 4, e6225.

61. Wang, J.; Wu, M.; Wang, B.; Han, Z.; Pest Manage. Sci. 2013, 69,7 .

Submitted: December 8, 2019

Published online: May 26, 2020 